\title{
Gas line observations of disks ${ }^{\star}$
}

\author{
Odysseas Dionatos ${ }^{a}$ \\ ${ }^{1}$ University of Vienna, Department of Astrophysics, Türkenschanzstrasse 17, 1180 Vienna, Austria
}

\begin{abstract}
Gas represents the bulk of the mass content in protoplanetary disks. It is therefore observations of gas that can provide important information of physical structure and chemical evolution of disks. This involves a detailed understanding of the spectroscopic signatures of the gaseous components and the conditions under which their excitation occurs. In the following I provide an introduction to the principles of molecular spectroscopy by discussing the spectral signatures of the most abundant molecular species found in protoplanetary disks. The presentation for each molecule is supplemented by an up-to-date discussion of relevant observations and subsequent constraints on the corresponding physical conditions in protoplanetary disks. A primer to excitation diagrams as a simple but effective tool for accessing the excitation of spectral lines is presented towards the end of the chapter.
\end{abstract}

\section{Introduction}

The gaseous content of a disk is confined within the limits of adsorption of molecules onto dust grains towards the mid-plane and their photo-dissociation towards the upper layers. The two limits that define the disk's atmosphere imply that its vertical extent may vary for different species. In addition the gaseous disks extends inwards beyond the dust sublimation radius, but may not be as extended as the dust component towards the outer parts of the disk. Excitation of the gas is expected to have a radial dependence on the disk; it is much more pronounced in the inner regions ( $R<10 \mathrm{AU})$, where temperatures and radiation field from the central star are enhanced but gradually drops towards the outer regions. An additional vertical dependence is implied by the density as discussed in the next chapter. A summary of the different excitation conditions traced by molecular species, as a function of radius and height in the disk is given in Fig. 1.

Gas in accretion disks rotates following a Keplerian motion so that the line broadening, when spectrally resolved, can provide constraints to the location of the excited gas in the disk. A characteristic Keplerian line-profile is shown in Fig. 2, and its interpretation is discussed in the following section.

\section{Characteristic line profiles}

The line-of-sight curves of gas moving at constant velocity in a keplerian-rotating disk at inclination $\theta$ are described by the equation

\footnotetext{
$\star 7^{\text {th }}$ Lecture of the Summer School "Protoplanetary Disks: Theory and Modelling Meet Observations"

a e-mail: odysseas.dionatos@univie.ac.at
} 


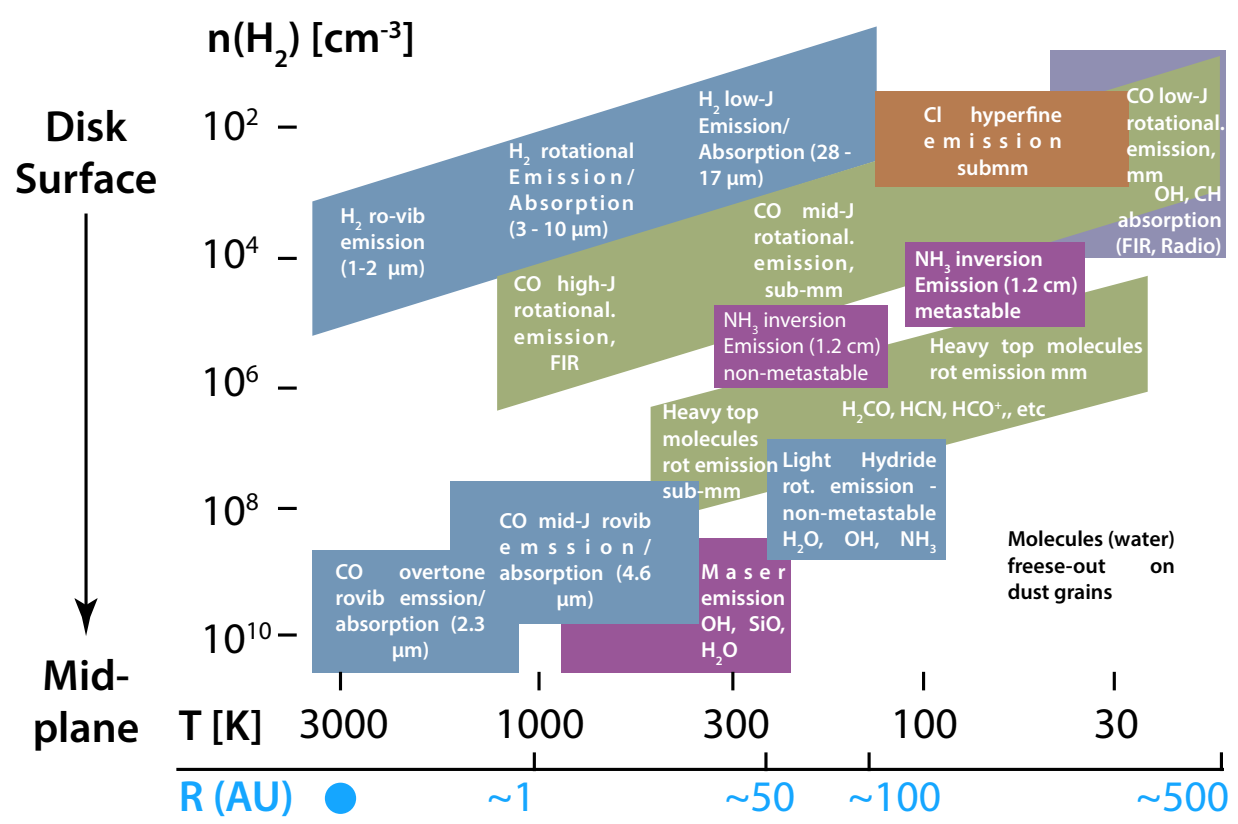

Figure 1. Schematic representation of the radial and vertical locations in a disk where molecular species are expected to dominate the line emission.

$$
R=R_{\text {out }}\left(\frac{u_{\text {out }}}{u}\right)^{2} \cos ^{2} \theta,
$$

where $u$ is the line-of-site velocity vector and $R_{\text {out }}$ and $u_{\text {out }}$ are the outer disk radius and the corresponding velocity at that limit. At the limit $u \rightarrow 0$ the iso-velocity contours move closer to the line-of-sight axis dissecting perpendicularly the disk and form the line minima. As long as the velocity remains smaller than $u_{\text {out }}$, the iso-velocity contours on the disk surface are open and truncated at $R_{\text {out }}$. The maximum contour occurs for $u=u_{\text {out }}$ that is the first closed (non-truncated) contour which contributes to the line peaks. Further increase in the velocity for $u>u_{\text {out }}$ results in shorter, closed contours lying symmetrically on the horizontal disk mid-line that move towards to the center of the disk and contribute to the outer line wings. The contribution of the different curves of constant velocity on the line profile becomes even more pronounced if velocity intervals corresponding to surface areas on the disk are considered, as shown in Fig. 2. The limiting $u_{\text {out }}$ can be derived from the separation of the line-peaks and Eq. 1 once $R_{\text {out }}$ and the inclination $\theta$ are known. An example of spectrally resolved disk line-profiles and spatially resolved velocity distribution around the debris disk of HD 21997 is provided in Fig. 2 (Kóspál et al. 2013).

Observations of double-peaked line-profiles which are characteristic of a Keplerian-rotating disk are very important as they provides information on the geometry and gas content of the disk. However detections of such lines is not always straightforward. In the case of still accreting disk-sources, disk emission is often masked by the much stronger line emission of shocked gas along the jets and outflows in a direction perpendicular to the disk. The double peaked profile can also be masked out in the case that the source is still surrounded by significant amounts of gas that renders it optically thick. But even in the case of high angular and spectral resolution, double-peaked profiles can come as a result of different projections and optical depth effects (Elitzur et al. 2012). 

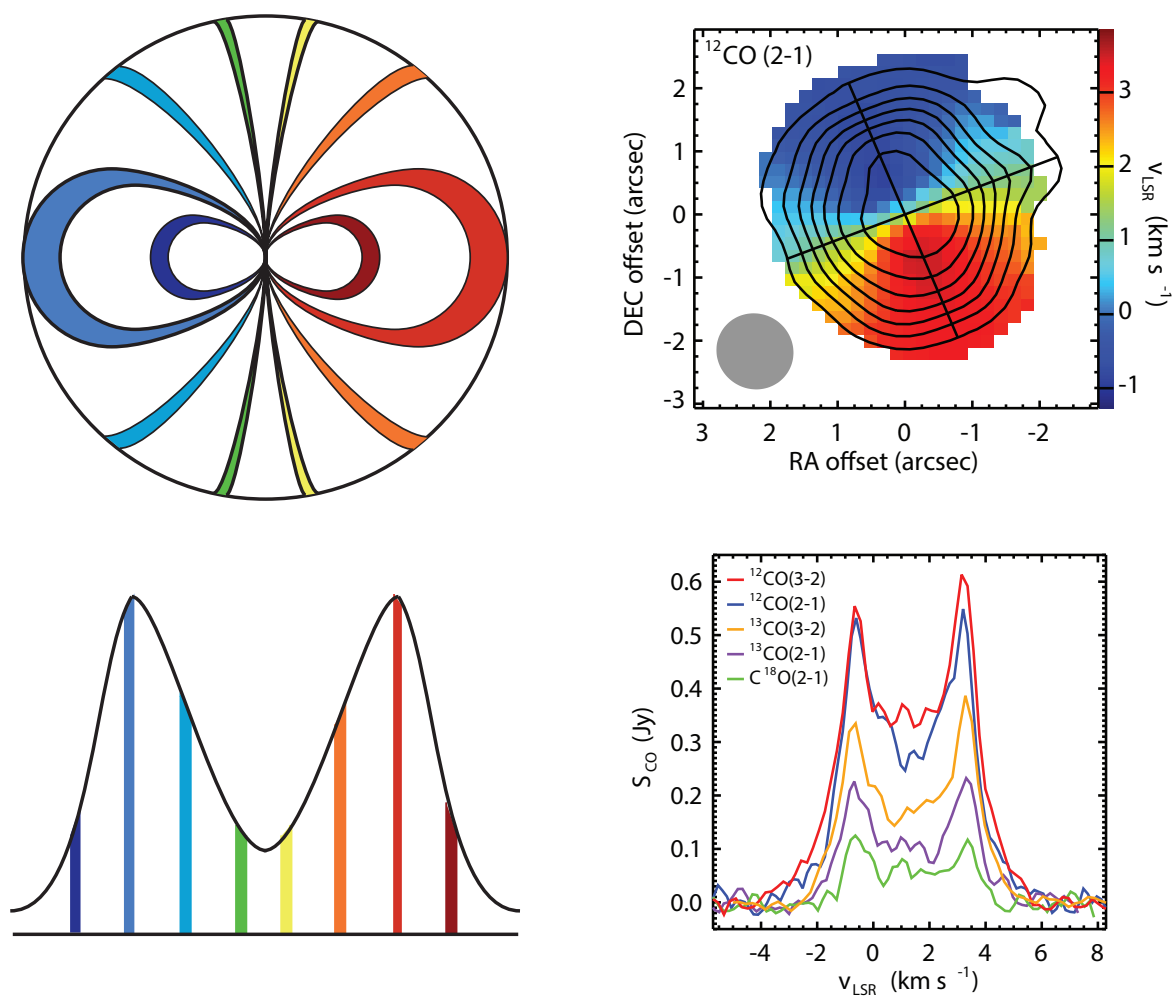

Figure 2. Left: Iso-velocity contours on a disk and their corresponding contributions in forming the characteristic Keplerian line-profile. Right: Resolved spectra for a series of CO lines and isotopologues toward HD 21997 and the corresponding velocity structure of the disk (from Kóspál et al. 2013, (c) AAS, reproduced with permission).

\section{Molecular transitions}

Nuclei bound in a molecule can undergo rotational, vibrational and bending motions. Such motions are quantized and transitions from one state to another result in photons usually occurring in the near-IR up to the millimeter regimes of the electromagnetic spectrum. In a similar way to atoms, molecules undergo also electronic transitions (discussed towards the end of this chapter), however the latter require higher excitation energies and hence such transitions produce photons in the optical and ultraviolet parts of the spectrum. Therefore the total excitation energy of a molecule will be given by the sum of the rotational, vibrational and electronic excitation energies:

$$
E_{\mathrm{tot}}=E_{\mathrm{rot}}+E_{\mathrm{vib}}+E_{\mathrm{el}} \quad \text { with } \quad E_{\mathrm{rot}}<<E_{\mathrm{vib}}<<E_{\mathrm{el}}
$$

Albeit the large separation between different transition energies, rotational states and transitions between them are constrained by the total energetic state of the molecule, which is dominated by the electronic configuration. The description of the electron configurations of molecules borrows from the atomic spectroscopic notation, however the spherical symmetry of atomic nuclei is not applicable in the case of molecules, but instead it depends on existing axes of symmetry between the atomic nuclei. In the case of diatomic molecules, the total electronic angular momentum $L$ is coupled to the 
internuclear axis, therefore its projection $\Lambda$ onto that axis is a conserved quantity. When $\Lambda \neq 0$ then $L$ can have two orientations onto the internuclear axis so that $\Lambda$ has a degeneracy of 2 . $\Lambda$ can take integer values of $0,1,2,3, \ldots$ which are assigned to the characters $\Sigma, \Pi, \Delta, \Phi, \ldots$. Thus the spectroscopic notation for the electronic states of molecules borrows from the formalism of multi-electron atoms $(\mathrm{S}, \mathrm{P}, \mathrm{D}, \mathrm{F}, \ldots)$ with the difference that in the case of molecules the symbols in use are the equivalent upper case characters of the Greek alphabet.

The total electron spin in a molecule is not strongly coupled to the nuclei, so the molecular electron spin follows the atomic notation. According to this prescription, the total electron spin angular momentum is $S$ and the electronic states are coined by their spin multiplicity $2 S+1$, given as a leading superscript to the $\Lambda$ state.

For the diatomic molecules described in the following sections, the ground state is a closed shell (i.e. ${ }^{1} \Sigma$ state). The only exception is $\mathrm{OH}$ that has an open shell ground state $\left({ }^{2} \Pi\right)$. This adds additional terms in the description of the energy levels due to the interaction of the electron spin angular and orbital momentum with the atomic nuclei.

\subsection{Rotational transitions of molecules}

Rotational transitions occur in certain electronic configurations where the molecule is considered to be a static system (Fig. 3). In principle, rotational transitions occur in molecules that have permanent dipole moment $(\mu)$. The strength of produced molecular lines is a function of $\mu^{2}$ so that molecules with large dipole moments have intense resonances. All heteronuclear diatomic molecules possess permanent dipole moments, but homonuclear molecules do not. In the latter case, molecules like $\mathrm{H}_{2}$ undergo electric quadrupole transitions that are substantially weaker. In the following section we discuss the rotational transitions of a homonuclear $\left(\mathrm{H}_{2}\right)$ and a heteronuclear molecule $(\mathrm{CO})$ that both have a closed electron shell configurations $\left({ }^{1} \Sigma\right)$.

\subsubsection{Closed electronic shell diatomic molecules: $\mathrm{H}_{2}, \mathrm{CO}$}

To a first degree, we describe the possible rotational states of a molecule assuming no vibrational motions. Level energies according to the rigid-rotor approximation are given by the relation:

$$
E=\frac{h^{2}}{8 \pi^{2} I} J(J+1)
$$

where $I$ is the moment of inertia at the center of mass of the system $\left(I=m_{\text {red }} r_{r e d}^{2}\right)$, and $J$ is the rotational quantum number of a state. The nuclei distances in a molecule typically measure a few Ångström so the moment of inertia between different molecules is dominated by the reduced mass of the nuclei. The constant terms for a molecule are conveniently wrapped in the rotational constant $B_{0}$, so that Eq. (3) is re-written as:

$$
\begin{array}{llll}
E=h B_{0} J(J+1) & \text { where } & B_{0}=\frac{h}{8 \pi^{2} I} & \text { (units in } \mathrm{Hz}) \\
\frac{E}{h c}=B_{0} J(J+1) & \text { where } & B_{0}=\frac{h}{8 \pi^{2} c I} & \text { (units in } \left.\mathrm{cm}^{-1}\right)
\end{array}
$$

Allowed electric dipole transitions occur at single steps along the energy ladder of a molecule with $\Delta J= \pm 1$. The transition frequency will then be:

$$
v(J \rightarrow J-1)=2 B_{0} J
$$


Summer School "Protoplanetary Disks: Theory and Modeling Meet Observations"

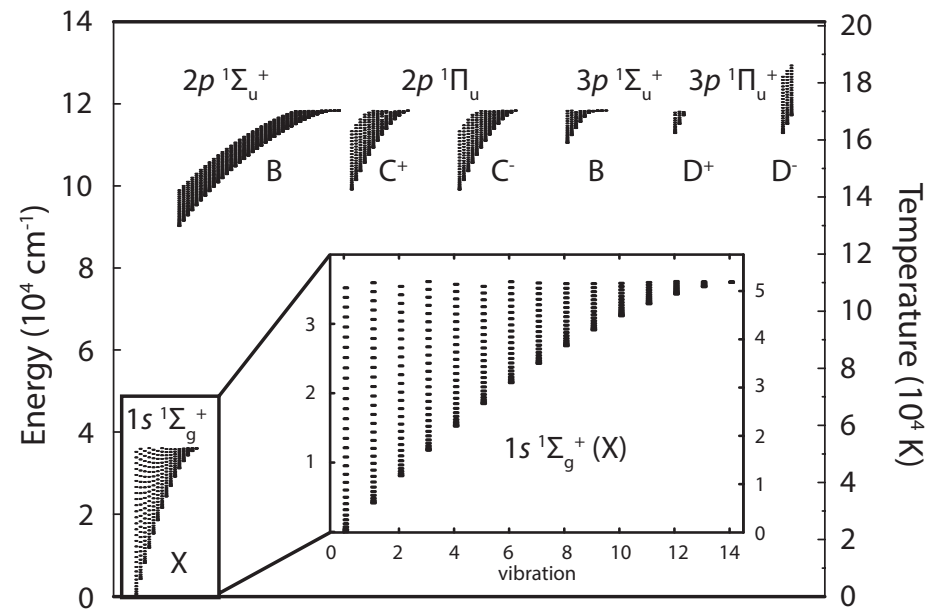

Figure 3. Energy levels for the $\mathrm{H}_{2}$ molecule for the ground and excited electronic states. To each electronic state corresponds a series of vibrational and rotational levels. The inset image provides a magnification of the ${ }^{1} \Sigma$ ground electronic state, where the individual vibrational levels forming "columns" and rotational levels within each vibrational level can be discriminated (adopted from Shaw et al. 2005, (c) AAS, reproduced with permission).

Taking $\mathrm{CO}$ as an example, the rotational constant is $B_{0} \sim 57 \mathrm{GHz}=1.9225 \mathrm{~cm}^{-1}$. Following Eq. (5) the expected rotational resonances will follow the prescription:

$$
v(J \rightarrow J-1)=J \cdot 115 \mathrm{GHz}
$$

For $\Sigma$ electron state diatomic molecules, electric dipole transitions follow the selection rule:

$$
\Delta J= \pm 1
$$

This gives rise to the $\mathrm{CO}$ rotational ladder, with the fundamental transition $(J=1 \rightarrow 0)$ at 115 $\mathrm{GHz}(2.6 \mathrm{~mm})$, which then for $(J=3 \rightarrow 2)(346 \mathrm{GHz}$ or $0.866 \mathrm{~mm})$ extends in the submillimeter. $\mathrm{CO}$ rotational lines up to $J=6 \rightarrow 5$ are therefore easily accessed from ground base facilities. In addition to the most common isotope $\left({ }^{12} \mathrm{C}^{16} \mathrm{O}\right), \mathrm{CO}$ comes into less abundant isotopologues ${ }^{13} \mathrm{C}^{16} \mathrm{O}$, ${ }^{12} \mathrm{C}^{17} \mathrm{O}$ and ${ }^{12} \mathrm{C}^{18} \mathrm{O}$. To a first approach, the exchange of an atom in a molecule with a heavier isotope results in increasing the reduced mass $\mu$ and subsequently the moment of inertia of the system which decreases the rotational constant $B_{0}$. This results in a closer spacing in the resulting resonances of heavier isotopologues but also to a significant shift in the overall resonances in comparison to the most abundant form of CO. The fundamental (1-0) frequency for ${ }^{13} \mathrm{C}^{16} \mathrm{O}$ lies at $2.72 \mathrm{~mm}$ and for ${ }^{12} \mathrm{C}^{17} \mathrm{O}$ at $2.67 \mathrm{~mm}$. Such differences are easily resolved with current instrumentation.

Electric dipole selection rules break down in the case of homonuclear molecules, which bear no permanent electric dipole moment. Such is the case of $\mathrm{H}_{2}$ for which the first allowed transitions are from electric quadrupole. In hydrogen, combination of two protons with spin $s=1 / 2$ results into two configurations for the total molecular spin $S=0$ or 1 , corresponding to the nuclear spins being anti-parallel or parallel. These two states of the $\mathrm{H}_{2}$ molecule, named ortho- $\mathrm{H}_{2}$ (parallel spin, $\mathrm{S}=1$ ) and para $-\mathrm{H}_{2}$ (anti-parallel spin, $\mathrm{S}=0$ ) are almost independent and changes from one state to the other occur only through proton exchange reactions with other species (e.g. $\mathrm{H}, \mathrm{H}^{+}, \mathrm{H}^{+3}$ ). Quantum mechanical analysis yields that the total wavefunction of the molecule must be anti-symmetric, so that if the nuclear spins are anti-parallel (parallel) the rotational quantum number $J$ must be even (odd). 
Selection rules for electric quadrupole transitions dictate that:

$$
\Delta J= \pm 2
$$

Being a very light molecule, $\mathrm{H}_{2}$ has a large rotational constant $B_{0}=1750 \mathrm{GHz}$ or $62 \mathrm{~cm}^{-1}$ which results in widely spaced rotational levels compared to heavier molecules. The lowest energy transition for $J=2 \rightarrow 0$ occurs at $28.2 \mu \mathrm{m}$ for the para $-\mathrm{H}_{2}$.

The spectroscopic description of rotational transitions for closed shell heteronuclear diatomic molecules such as $\mathrm{CO}$ is straightforward and has been already used in the previous discussion. Transitions are designated by the number of the upper and the lower rotational levels, using in between an appropriate separator (most commonly "'-' or “ $\rightarrow$ "). For the case of $\mathrm{H}_{2}, \Delta J$ jumps of +2 and -2 are described by the letters $\mathrm{S}$ and $\mathrm{O}$ respectively, followed by the $J$ number of the lower state into parenthesis. For example, the $J=2 \rightarrow 0$ fundamental rotational transition is coined as $S(0)$, and the $J=3 \rightarrow 1$ ortho- $\mathrm{H}_{2}$ transition as $S(1)$.

$\mathrm{H}_{2}$ holds the keys to access the total gas content in disks. It is by far the most abundant molecule in star-forming regions and its electric quadrupole transitions ensure that the emission is optically thin up to high densities. In addition, $\mathrm{H}_{2}$ freezes out onto dust grains at very low temperatures and becomes easily thermalized, so that it can trace the gas content deep into the disk mid-plane and provide means for direct gas temperature measurements. However the high rotational constant means that even the lowest energy levels require high temperatures $(\sim 300 \mathrm{~K})$ to be collisionally populated. Early efforts to trace $\mathrm{H}_{2}$ in protostellar disks (Thi et al. 2001) were later disputed on basis that the detected emission is originating from shocked gas in jets. In a large survey of protostellar disks with Spitzer, Lahuis et al. (2007) reported no traces of the two lowest transitions at $28.2 \mu \mathrm{m}$ and $17.0 \mu \mathrm{m}$, but detections of the two subsequent $\mathrm{H}_{2}$ lines at $12.3 \mu \mathrm{m}$ and $9.7 \mu \mathrm{m}$ at $\sim 8 \%$ of the examined sources, rising from a gas at an estimated temperature of $\sim 100-200 \mathrm{~K}$. The derived gas mass from the warm $\mathrm{H}_{2}$ gas is very low, corresponding to a few Jovian masses.

The potential and significance of $\mathrm{H}_{2}$ in accessing the disk mass has been recently demonstrated with hydrogen deuteride (HD). Examining the disk around the T-Tauri star TW Hya, Bergin et al. (2013) reported on the detection of 4 HD transitions (Fig. 4) which allowed them to set a lower limit
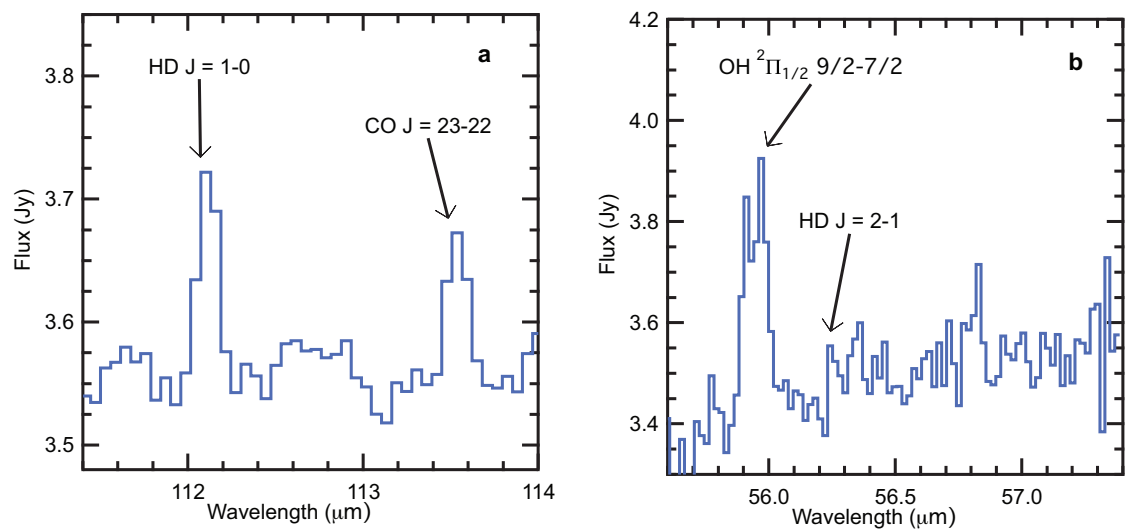

Figure 4. Detections of HD lines in the disk of TW Hya that made it for the first time possible to assess its total mass. Figure reprinted by permission from Macmillan Publishers Ltd: Nature (Bergin et al. 2013), copyright (2013). 
on the disk mass of $0.05 \mathrm{M}_{\odot}$. This remains however a unique case; the detection of HD is nontrivial as its abundance is very low and the lowest energy transitions fall in the far-infrared, requiring space-born facilities to be observed.

For deriving the total gas content in disks we rely on indirect methods. Commonly, the total gas is either estimated from the dust content, or from observations of $\mathrm{CO}$. The lower rotational transitions of $\mathrm{CO}$ are easily accessible from ground-based facilities and its isotopologues ${ }^{13} \mathrm{CO}$ and $\mathrm{C}^{18} \mathrm{O}$ provide probes for constraining the optical depth and hence the disk surface density. Such techniques applied onto high angular resolution observations lead to a detailed description of the radial and vertical gas content of disks. (e.g. Piétu et al. 2007; Rosenfeld et al. 2013). CO emission arises from a warm layer at an intermediate hight in the disk; above a certain level $\mathrm{CO}$ is photo-dissociated, and below that towards the disk mid-plane it freezes out onto dust grains $(T \approx 20 \mathrm{~K})$. Due to this characteristics $\mathrm{CO}$ probes only the certain layer that it survives in gaseous form between the dissociation and freeze-out boundaries. Another limitation comes from the fact that $\mathrm{CO}$ lower $J$ transitions are easily excited even at low temperatures and are optically thick. It is therefore often that diffuse foreground emission masks the emission from the disks and renders it challenging to obtain reliable measurements. In order to overcome the limitations imposed by CO, Guilloteau et al. (2014) have recently proposed the use of $\mathrm{CN}$ as a more reliable probe of the gas content of disks.

\subsubsection{Open electronic shell diatomic molecules: $\mathrm{OH}$}
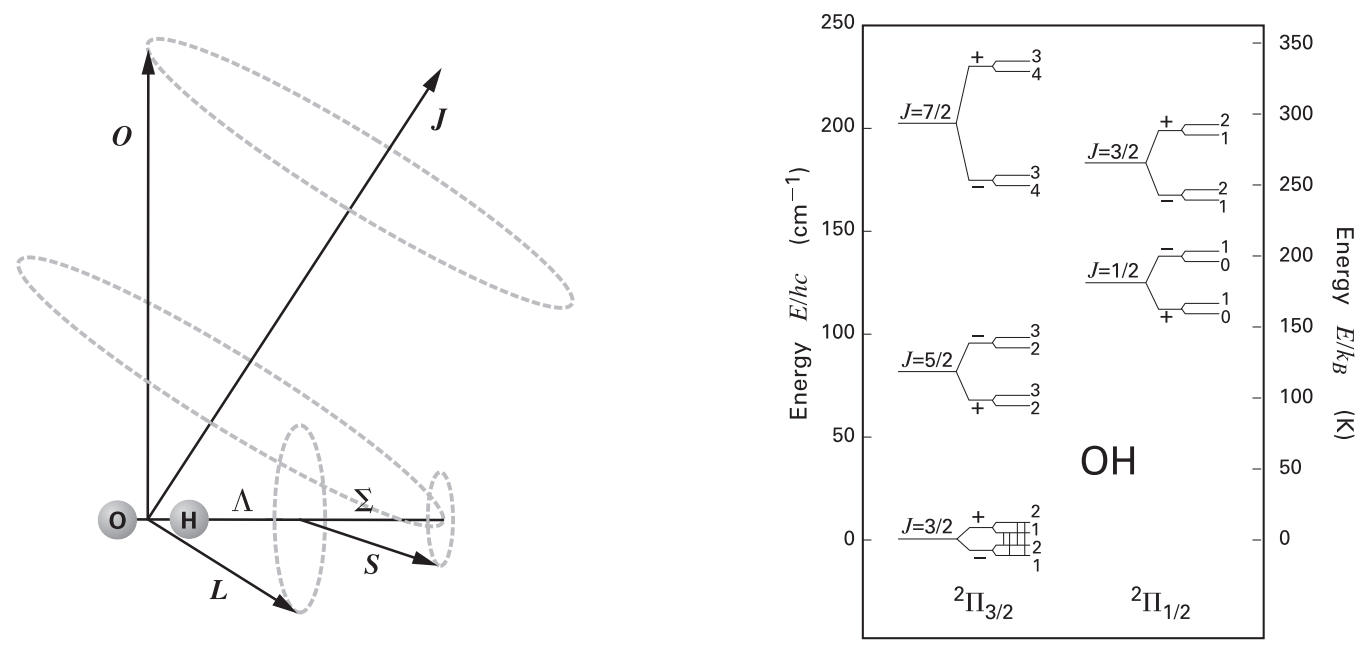

Figure 5. Left: Schematic representation of the different interacting components that define the $\mathrm{OH}$ rotational levels. The unpaired electron orbital and spin angular momentum vectors $L$ and $S$ have projections onto the internuclear axis $\Lambda$ and $\Sigma$, respectively. The nuclear angular momentum $O$ precesses around the total angular momentum J. Right: Rotational energy levels of the $\mathrm{OH}$ molecule. The two ladders correspond to different orientations of the unpaired electron spin. Each level is initially split due to $\Lambda$-doubling and further split due to unpaired electron's spin coupling (from Stahler \& Palla 2004).

Unpaired electrons induce a substructure to the rotational levels of a molecule, which rises from the electric and magnetic field of the open shell electrons. Free radicals in their electronic ground states but also also closed-shell molecules in excited electronic states may fall in this category. In the case of 
$\mathrm{OH}$, both electronic orbital and electronic spin angular momentum are present, and coupling of these spins to the rotational states of the molecule leads in hyper-fine splitting of the latter.

The projection of the electronic orbital angular momentum vector $L$ onto the internuclear axis is $\Lambda$ (Fig. 5). Similarly, the component of the electronic spin vector $L$ onto the internuclear axis is defined as $\Sigma$. The precession of $L$ around the internuclear axis leads to fixed values of $\Lambda$, where $|\Lambda|=0,1,2$, $3 \ldots$ is designated with the letters $\Sigma, \Pi, \Delta, \Phi$ etc. The electronic spin is labeled at the top left of $\Lambda$ in terms of $2 S+1$, so that a ${ }^{2} \Pi$ state stands for $\Lambda=1, S=1 / 2$.

The component of the total electron angular momentum along the internuclear axis is denoted as $\Omega$ and given by $\Omega=\Lambda+\Sigma$. For a molecule in an ${ }^{2} \Pi$ electronic state the values for the projection of possible quantum numbers are given in the following Eq. (9).

$$
\begin{array}{lll}
\Lambda=+1 & \Sigma=+\frac{1}{2} & \Omega=+\frac{3}{2} \\
\Lambda=-1 & \Sigma=-\frac{1}{2} & \Omega=-\frac{3}{2} \\
\Lambda=+1 & \Sigma=-\frac{1}{2} & \Omega=+\frac{1}{2} \\
\Lambda=-1 & \Sigma=+\frac{1}{2} & \Omega=-\frac{1}{2}
\end{array}
$$

The two possible orientations of $\Lambda$ give rise to the $\Lambda$-doubling. The electron spin coupling gives rise to an additional separation of the rotational levels into fine structure states as shown in Fig. 5. The value of $|\Omega|$ is written as a lower-right subscript in the state label. In the current example, a ${ }^{2} \Pi$ molecule has two ${ }^{2} \Pi_{3 / 2}$ and ${ }^{2} \Pi_{1 / 2}$ fine structure states for each $\Lambda$ component. If $\Omega$ is negative, then the ${ }^{2} \Pi_{3 / 2}$ state lies at lower energy than the ${ }^{2} \Pi_{1 / 2}$ and in that case the doublet is called inverted. Such is the case for $\mathrm{OH}$ that has a ${ }^{2} \Pi_{3 / 2}$ ground state.

The rotational energy taking into account the $\Lambda$-doubling and spin degeneracy is given by:

$$
E_{\text {rot }}=B\left[J(J+1)+S(S+1)+2 \Lambda \Sigma+\Lambda^{2}-2 \Omega^{2}\right]
$$

So the rotational levels in the case of $\mathrm{OH}$ are characterized by the rotational quantum number $J$ and the coupling of the rotational motion with the electronic orbital angular momentum and electronic spin. Transitions can occur between rotational levels which result in lines in the Far-IR domain, but also between the $\Lambda$-doubling components which produce lines in the mm parts of the spectrum. The spectroscopic notation for rotational transitions of $\mathrm{OH}$ are rather complex, as it requires a complete description of the levels involved (e.g. the far-IR line ${ }^{2} \Pi_{1 / 2}-{ }^{2} \Pi_{1 / 2}, 7 / 2^{-}-5 / 2^{+}$at $71.17 \mu \mathrm{m}$ ). OH is chemically related to water. The observational constraints derived from $\mathrm{OH}$ are discussed in the following section.

\subsubsection{Non-linear molecules: $\mathrm{H}_{2} \mathrm{O}$}

The general description of non-linear molecules requires cartesian projection of the total moment of inertia onto 3 axis. The principal moments of inertia are thus arbitrarily defined as $I_{a}, I_{b}$ and $I_{c}$ with the constraint that $I_{a}>I_{b}>I_{c}$. Molecules with axis of symmetry in rotation are called symmetric tops and are divided into prolate tops where $I_{a}>I_{b}=I_{c}$ and oblate tops for $I_{a}=I_{b}>I_{c}$. The energy levels of the rotational states for prolate and oblate tops are given by:

$$
\begin{aligned}
E_{\text {prolate }} & =\frac{h^{2}}{8 \pi^{2} I_{b}} J(J+1)+\frac{h^{2}}{8 \pi^{2}}\left(\frac{1}{I_{c}}-\frac{1}{I_{b}}\right) K^{2}=B J(J+1)+(C-B) K^{2} \\
E_{\text {oblate }} & =\frac{h^{2}}{8 \pi^{2} I_{b}} J(J+1)+\frac{h^{2}}{8 \pi^{2}}\left(\frac{1}{I_{a}}-\frac{1}{I_{b}}\right) K^{2}=B J(J+1)+(A-B) K^{2}
\end{aligned}
$$


Water molecule is an asymmetric top, having three unequal moments of inertia along its principal axis. This means that the total energy and angular momentum are conserved, but not the projection of the latter along any axis of symmetry of the molecule. The spectroscopic notation of water borrows from the symmetric tops and rotational states are labeled as $J_{K_{c}}, K_{a}$, where $K_{c}$ and $K_{a}$ subscripts denote exchange of principle axis $\mathrm{B}$ to $\mathrm{C}$ for prolate and $\mathrm{A}$ for oblate symmetric top forms. Electric dipole selection rules dictate that $J=0, \pm 1$ and $K_{a}, K_{c}$ can change by $\pm 1, \pm 3$. In addition, water, like molecular hydrogen, comes into ortho and para forms owing to the hydrogen spin nuclei being orientated parallel or anti-parallel, respectively.
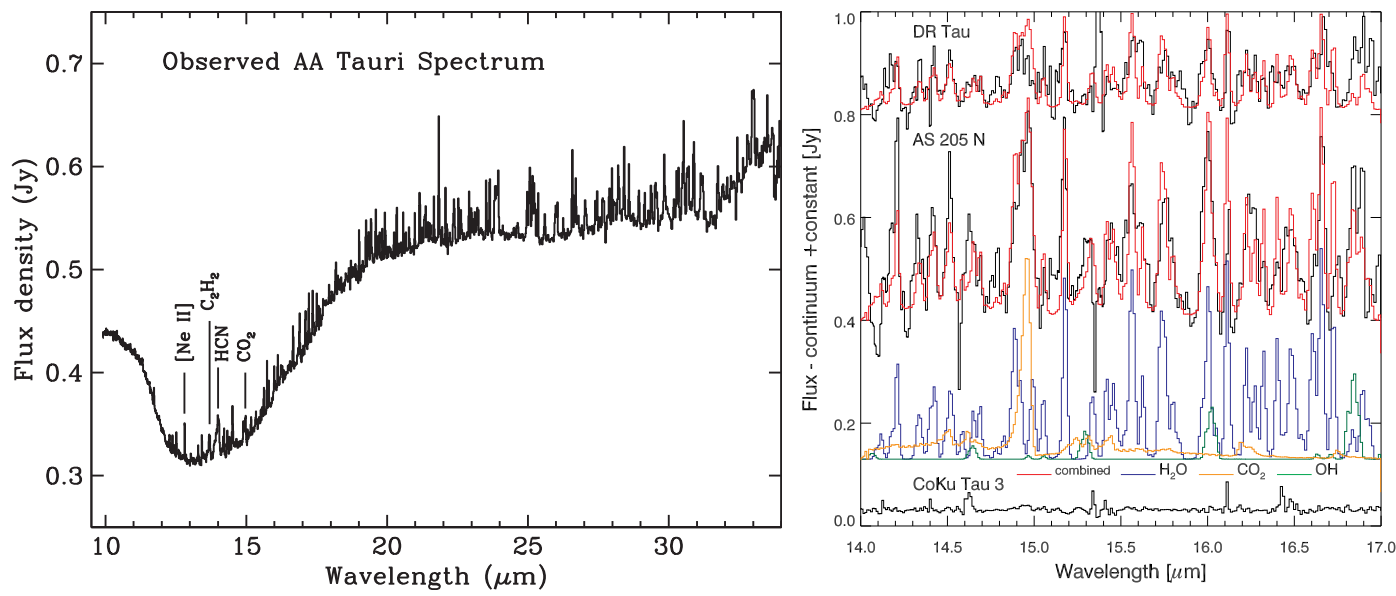

Figure 6. Spitzer mid-IR observations of $\mathrm{H}_{2} \mathrm{O}$ and $\mathrm{OH}$ "forest" of lines originating in the terrestrial planet forming regions of disks. Left: Figure from Carr \& Najita (2008, reprinted with permission from AAAS). Right: Figure from Salyk et al. (2008, (c) AAS, reproduced with permission)

Water has a large number of allowed rotational transitions at far-infrared and millimeter wavelengths and when excited, it provides an important cooling mechanism for the atmospheres of disks. Water lines are hardly reached with ground-based facilities, as the atmospheric water vapor content attenuates any propagating signal.

High excitation lines from $\mathrm{H}_{2} \mathrm{O}$ and $\mathrm{OH}$ dominate, in some cases, the mid-IR spectrum. Such high excitation lines that trace gas at temperatures of $500-1000 \mathrm{~K}$ are expected to be excited in the inner 1 - 5 AU of the disk (Carr \& Najita 2008; Salyk et al. 2008). This region corresponds to the terrestrial planet-forming region so such observations have an important impact on the formation theories of planetary atmospheres. $\mathrm{H}_{2} \mathrm{O}$ and $\mathrm{OH}$ lines at lower temperatures $(100-300 \mathrm{~K})$ have been observed in the far-IR with Herschel (Fedele et al. 2013). Such lines are believed to probe gas at larger distances in the disk ( $>20 \mathrm{AU}$ ). $\mathrm{The} \mathrm{OH} / \mathrm{H}_{2} \mathrm{O}$ line ratio from these observations is consistent with a radially decreasing UV field.

\subsection{Vibrational transitions of molecules}

The description of the rotational transitions of molecules was based on the approximation that the molecule behaves as a rigid body so that the internuclear distances remain fixed. For the description 
of vibrational motions within a molecule, the quantum mechanical solution for the energy levels of the harmonic oscillator are given by:

$$
E_{v}=\hbar \omega\left(v+\frac{1}{2}\right)
$$

where the quantum number $v=0,1,2, \ldots$ and $\omega$ is the angular frequency. In spectroscopic jargon, $\hbar \omega$ is given in wavenumber units of $\mathrm{cm}^{-1}$. In principle any transition $\Delta v$ between two vibrational states is allowed, even between different electronic states, however electric dipole transitions that $\Delta v= \pm 1$ are in principle much stronger. For electric dipole transitions:

$$
\Delta E_{v}=\hbar \omega\left(v+1+\frac{1}{2}\right)-\hbar \omega\left(v+\frac{1}{2}\right)=\hbar \omega
$$

where $\omega$ is the fundamental frequency. Vibrational transitions often occur with a simultaneous change

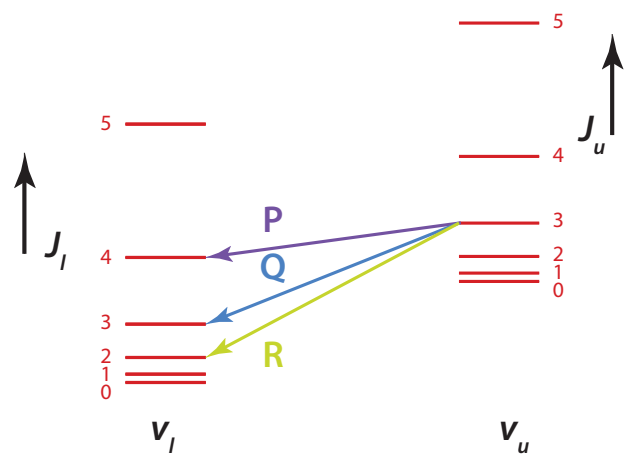

Figure 7. Schematic representation of the $R-, P-$ and $Q$-branch transitions between an upper and lower level vibrational states $v_{u}$ and $v_{l}$.

in the rotational state, thus the notion ro-vibrational transitions. Allowed electric dipole transitions between rotational states dictate that $\Delta J=0, \pm 1$ but $J=0 \leftrightarrow 0$ transitions are now allowed. For molecules in $\Sigma$ electron configurations $\Delta J= \pm 1$. In the case of homonuclear molecules (e.g. $\left.\mathrm{H}_{2}\right)$ having no permanent electric dipole moment, the allowed electric quadrupole transitions dictate that $\Delta J=0, \pm 2$, but no $J=0 \leftrightarrow 0$.

According to the electric dipole selection rules, ro-vibrational transitions between an upper $\left(J_{u}\right)$ and lower $\left(J_{l}\right)$ rotational state are classified in branches as

- $P$-branch: $\Delta J=-1, J_{u}=J_{l}-1$,

- $Q$-branch: $\Delta J=0, J_{u}=J_{l}$,

- $R$-branch: $\Delta J=+1, J_{u}=J_{l}+1$.

The $P$ and $R$ stand for the words poor and rich respectively, owing their names to the early days of molecular spectroscopy (Herzberg 1950). The $Q$-branch lines would lie in a rigid-rotor model at the same frequency between the $P$ and $R$ branches, hence the letter $\mathrm{Q}$ lying between $\mathrm{P}$ and $\mathrm{R}$ is used for their description.

The rotational constant between ro-vibrational states does not remain constant as the internuclear distance is prone to change. Increased distance at an upper state would result in increase of the 
Summer School "Protoplanetary Disks: Theory and Modeling Meet Observations"

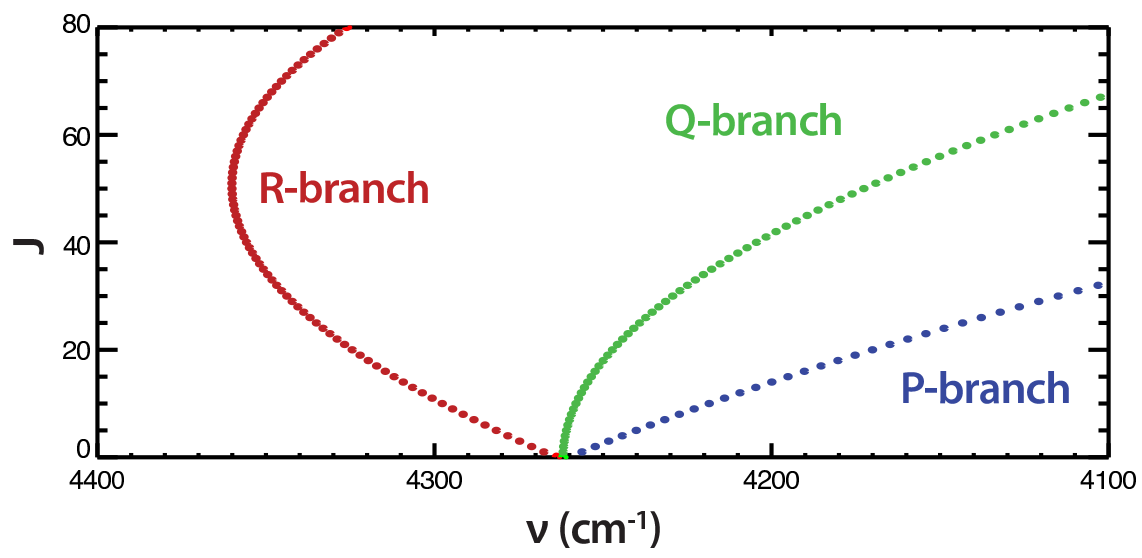

Figure 8. Fortrait diagram of $\mathrm{CO}$, showing the distribution of frequencies for transitions on the 3 branches, as a function of $J$. The characteristic $R$-branch bandhead becomes readily apparent.

moment of inertia of the system and decrease of the rotational constant, so that $B u<B l$. For the simplest case of a diatomic molecule, the energy change corresponding to a ro-vibrational transition from an upper level state described by the quantum numbers $v_{u}, J_{u}$ to a lower state $\left(v_{l}, J_{l}\right)$ is given by

$$
\Delta E\left(v_{u}, J_{u}, v_{l}, J_{l}\right)=\Delta E\left(v_{u}, v_{l}\right)+B_{u} J_{u}\left(J_{u}+1\right)-B_{l} J_{l}\left(J_{l}+1\right) .
$$

Substituting the values of $J_{u}$ with $J_{l}$ according to the different branches and setting $J_{l}=J$, equation 14 becomes

$$
\begin{aligned}
& \Delta E(J)_{P}=E_{0}-\left(B_{u}+B_{l}\right) J+\left(B_{u}-B_{l}\right) J^{2} \\
& \Delta E(J)_{Q}=E_{0}-\left(B_{u}-B_{l}\right) J(J+1) \\
& \Delta E(J)_{R}=E_{0}+2 B_{u}+\left(3 B_{u}-B_{l}\right) J+\left(B_{u}-B_{l}\right) J^{2}
\end{aligned}
$$

It becomes immediately apparent that there is a quadratic dependence of $\Delta E$ with $J$ for the $P$ and $R$ branches. Figure 8 displays a Fortrait diagram, showing the frequency for transitions in the different branches versus $J$. $P$-branch resonances start at the fundamental frequency $v_{0}$ and decrease monotonically as a function of frequency. $Q$-branch lines start also at $v_{0}$ and crowd around this point, slowly decreasing with increasing $J$. $R$-branch lines start at $2 B_{u}$ above $v_{0}$ and increase as a function of frequency for low values of $J$. However for

$$
J_{b}=\frac{3 B_{u}-B_{l}}{2\left(B_{u}-B_{l}\right)}
$$

the quadratic term in $J$ becomes dominant and for $J>J_{b}, R$-branch lines decrease as a function of frequency. Transitions crowd around $J_{b}$ creating a stationary point termed as bandhead.

For CO, the $R$-branch bandhead occurs for $J \sim 50$. Despite that the individual lines are not expected to be very strong for such high $J$ transitions, the cumulative effect of so many lines crowding around the bandhead renders them observable (Fig. 9). Such emission comes from the innermost regions of the disk $(0.3-3 \mathrm{AU})$ and traces gas at high temperatures and densities $(\mathrm{T}>2000 \mathrm{~K}$, $\mathrm{n}>10^{10} \mathrm{~cm}^{-3}$ ). Modeling of the bandhead emission also reveals that the velocity profiles of the lines kernel must be broad (e.g. Bik \& Thi 2004).

In the case of homonuclear molecules (e.g. $\mathrm{H}_{2}$ ), selection rules for vibrational transitions and electric quadrupole transitions are:

$$
\Delta J=0, \pm 2
$$



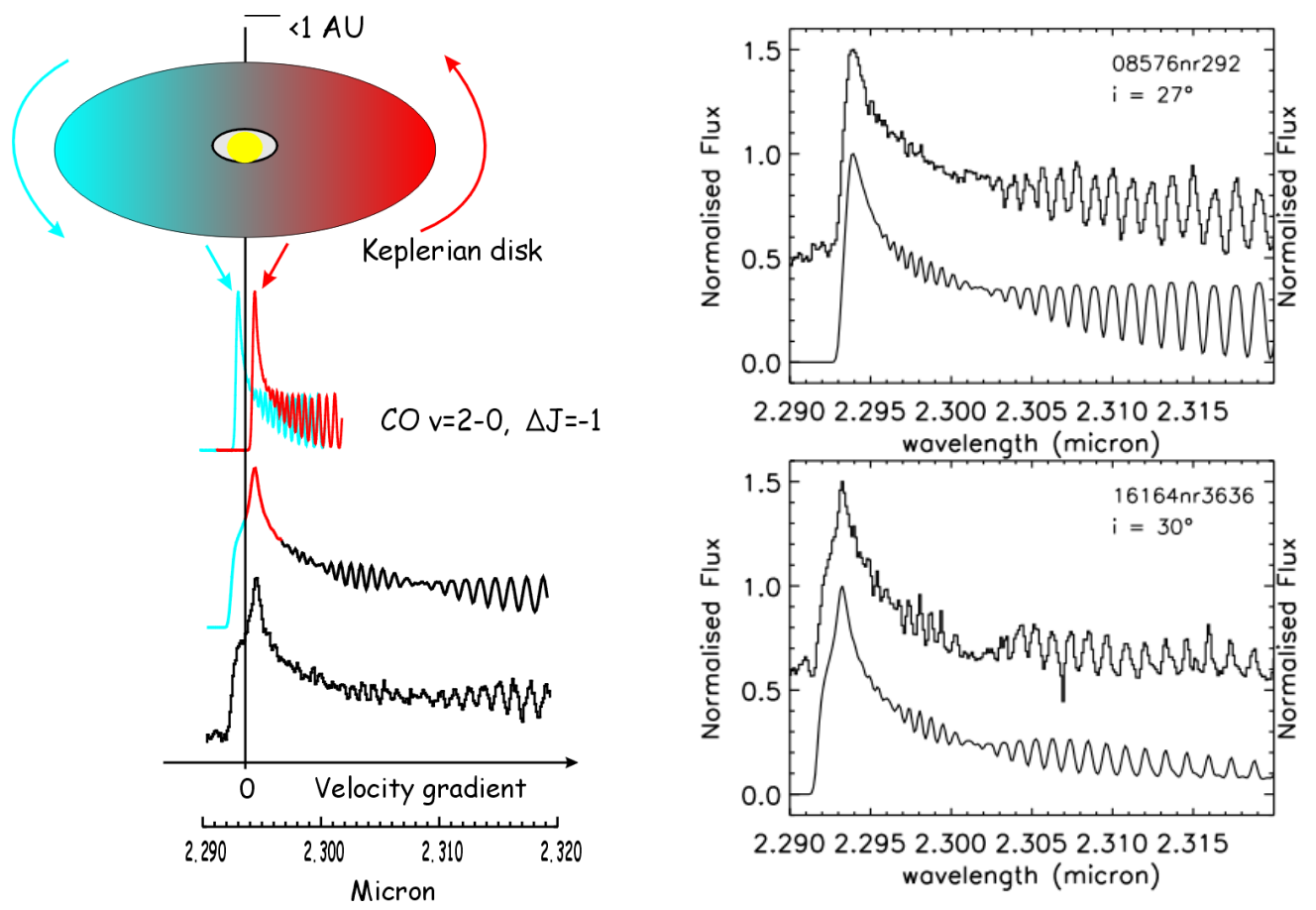

Figure 9. Left: The blue- and red-shifted line emission due to the rotation of the disk will result in superposition of the bandhead emission resulting in a "fringing" pattern. (W.F. Thi, private communication). Right: Observed and modeled spectra (upper and lower in each panel) of the CO $v=2-0$ bandhead emission from a high mass young stellar object (from Bik \& Thi 2004, reproduced with permission (c) ESO).

The general spectroscopic notation for ro-vibrational transitions requires the description of the upper and lower vibrational and rotational levels. It follows the general prescription:

$$
v_{\text {upper }}-v_{\text {lower }}, \Xi\left(J_{\text {lower }}\right)
$$

where $v_{\text {upper }}, v_{\text {lower }}$ can take any integer value and $\Xi$ is one of the letters corresponding in the $\Delta J$ jump as described in the following table.

\begin{tabular}{l|ccccc}
\hline$\Xi$ & $\mathrm{O}$ & $\mathrm{P}$ & $\mathrm{Q}$ & $\mathrm{R}$ & $\mathrm{S}$ \\
$\Delta J$ & -2 & -1 & 0 & 1 & 2 \\
\hline
\end{tabular}

Ro-vibrational lines from $\mathrm{CO}$ and $\mathrm{H}_{2}$ are often observed in disks. the $\mathrm{CO}$ fundamental (v=1-0) emission requires lower temperatures and densities than the bandhead emission and is therefore expected to trace gas at larger distances (1-2 AU) in the disk. The line velocity profiles, however, indicate that the $\mathrm{CO}$ emission may come from distances as small as $0.04 \mathrm{AU}$ in the case of disks around classical T-Tauri stars (CTTS), providing evidence that the gas disk extends further inwards than the dust sublimation radius of the disk (e.g. van der Plas et al. 2009). Observations of the $\mathrm{H}_{2}$ ro-vibrational transition at $2.12 \mu \mathrm{m}$ in a Herbig Ae/Be system (Carmona et al. 2011) display narrow velocity profiles indicating their location at radii of $\sim 10 \mathrm{AU}$. However the collisional excitation of the $\mathrm{H}_{2}$ transitions requires temperatures of at least $1000 \mathrm{~K}$ which are not expected to occur at such radii. It has consequently been proposed that the $\mathrm{H}_{2}$ is excited by X-ray and/or FUV radiation (Carmona et al. 2011). 


\subsection{Excitation diagrams as gas diagnostics in disks.}

The interpretation of line excitation and radiative transfer in disks is complex, see chapter by Kamp (2015a), and is best performed using specialized models. It is possible however, in particular cases, to extract valuable information directly from the data, given that a couple of requirements are fulfilled.

We start from the fundamental equation of radiative transfer

$$
\frac{d I_{v}}{d s}=-\kappa_{v} I_{v}+\epsilon_{v},
$$

where $\kappa_{v}, \epsilon_{v}$ are the frequency-dependent absorption and emission coefficients, respectively, for the gas and dust mixture, and $I_{v}$ is the spectral intensity. The solution for the Eq. (19), after continuum subtraction and frequency integration, in the optically thin limit, is

$$
I_{\text {line }}=\iint \epsilon_{v}^{i j} d v d s=\frac{h v_{i j}}{4 \pi} A_{i j} \int n_{i} d s
$$

where $I_{\text {line }}$ is the frequency-integrated line intensity, $\epsilon_{v}^{i j}$ is the line emission coefficient, $v_{i j}$ the line center frequency and $A_{i j}$ the Einstein coefficient for spontaneous emission. In the LTE limit (for densities well above the critical density) the fractional population of a level is described by the Boltzmann distribution

$$
\frac{N_{i}}{N_{t o t}}=\frac{g_{i}}{Z} \exp \left(-\frac{E_{i}}{k T}\right)
$$

where $N=n \int d s$ is the column density, $T$ the temperature, and $g_{i}$ is the statistical weight of upper level level $i$ taken as the product of the total nuclear spin and rotational degeneracies $g_{i}=(2 S+1)(2 J+1)$. The partition function $Z$ is given by the relation

$$
Z=\sum_{i} g_{i} \exp \left(-\frac{E_{i}}{k T}\right) .
$$

Combining Eqs. (20) and (21) yields the basic equation used in excitation diagrams

$$
\ln \left(\frac{N_{i}}{g_{i}}\right)=\ln \left(\frac{4 \pi I_{\text {line }}}{h v_{i j} g_{i} A_{i j}}\right)=-\frac{E_{i}}{k T}+\ln \left(\frac{N_{t o t}}{Z}\right) .
$$

Typically, excitation diagrams are constructed by plotting $\ln \left(N_{i} / g_{i}\right)$ versus $E_{i}$, however the first component is observationally determined by the $\ln \left(4 \pi I_{\text {line }} /\left(h v_{i j} g_{i} A_{i}\right)\right)$ term. In principle, if assumptions on LTE, optically thin medium and thermalization are fulfilled, then the observed points should fall on a straight line, the inverse slope of which gives the temperature $T$.

At the limit $E_{i}=0$, Eq. (23) becomes

$$
\ln \left(\frac{N_{i}}{g_{i}}\right)=\ln \left(\frac{4 \pi I_{\text {line }}}{h v_{i j} g_{i} A_{i j}}\right)=\ln \left(\frac{N_{t o t}}{Z}\right)
$$

and, therefore, the total column density can be derived from the intercept, given that the partition function $Z$ is known. Partition functions for simple molecules can be calculated analytically from equation 22 , however one has to bear in mind that sums have to act over a significant number of energy levels.

Interpretation of deviations from linearity in excitation diagrams can be complex. In principle lines at different excitation energies may trace the gas component at different radii in a disk. In that 

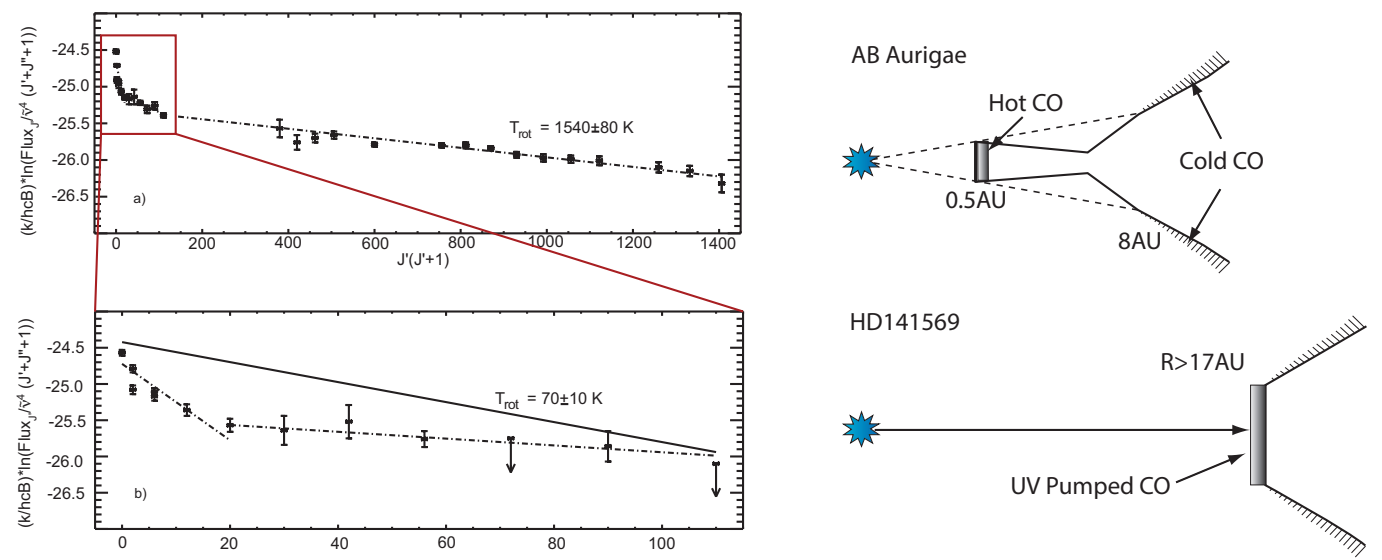

Figure 10. Excitation diagrams for the mid-IR CO ro-vibrational lines observed towards the HAeBe star AB Aur. The lower panel shows a magnification of the leftmost area of the top panel, where the distribution of observed line intensities deviates from linearity due to optical depth effects. The sketch on the right panel indicates the possible origin of the hot and cold CO emission (from Brittain et al. 2003, (c) AAS, reproduced with permission).

case, multiple temperature components can be fit with piece-wise linear function. Lines coming from electric quadrupole transitions (e.g. $\mathrm{H}_{2}$ ) are expected to be optically thin, however this may not be the case for other molecules. Optical depth can vary as a function of transition energy so that lower energy transitions of a molecule can be optically thick while higher energy levels may remain optically thin, resulting in mimicking a two-temperature component pattern in excitation diagrams (see Fig. 10), see more detailed dicussion in the chapter by Kamp (2015b). Similar patterns may appear in the case that the density is below the critical density and thus the gas is not thermalized. A detailed discussion on the applicability and effects of excitation diagrams can be found in Goldsmith \& Langer (1999).

\subsection{Molecular electronic transitions}

Electron transitions in molecules require high energies, that in most cases could lead to the dissociation of a molecule. It is therefore that excitation of electron states of molecules does not occur through collisions but rather by non-thermal (radiative) processes. Lines from electronic transitions lie at the same wavelength range as the electronic transitions of atoms, which is the visible and ultraviolet. The description of electronic transitions in molecules are more complex as one needs to take into account the corresponding changes in the electronic, vibrational and rotational states for each possible transition. In the current discussion we will only consider electric dipole transitions in diatomic molecules. Selection rules for such transitions are presented in Table 1

The selection rules on rotational and vibrational motions have been already discussed in the corresponding sections. In addition to these, electronic transitions require that the total electron spin is conserved. The selection rule on the total orbital angular momentum dictate that electronic transitions can occur between adjacent electronic or the same electronic states (e.g. $\Sigma-\Sigma, \Sigma-\Pi$ ). The last selection rules dictate change of symmetry under electric dipole transitions.

$\mathrm{H}_{2}$ electronic transitions due to fluorescence have been observed in the disk of TW Hya (Herczeg et al. 2004). In UV fluorescence, high energy photons photons excite $\mathrm{H}_{2}$ from the ground $\Sigma^{+}$to the $\Pi$ electronic state (Fig. 11). De-excitation occurs through a cascade from the excited state to an excited 
Summer School "Protoplanetary Disks: Theory and Modeling Meet Observations"

Table 1. Selection rules for electric dipole transitions for diatomic molecules (from Tennyson 2005)

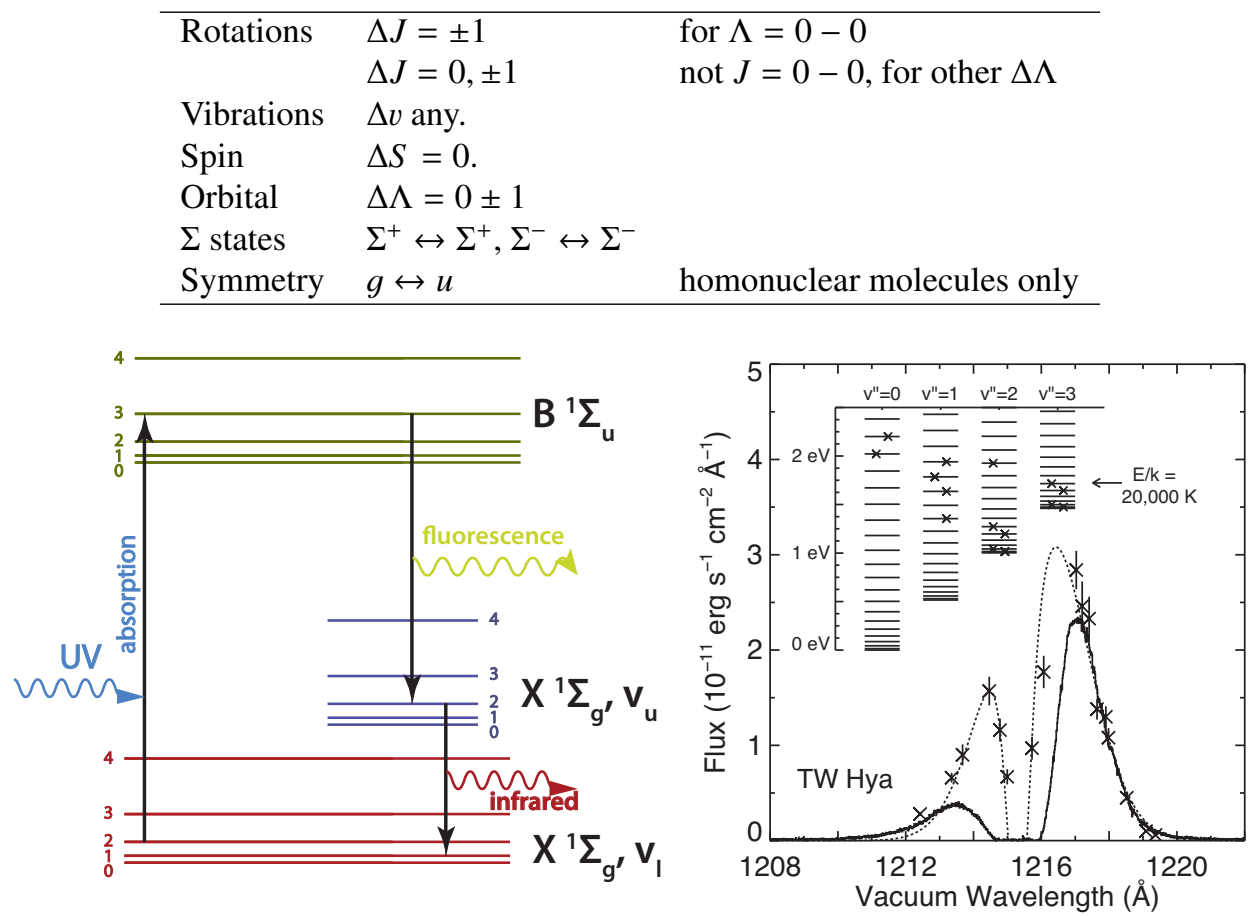

Figure 11. Left: Schematic representation of $\mathrm{H}_{2}$ fluorescence: an incoming UV photon excites $\mathrm{H}_{2}$ from the ground $X^{1} \Sigma_{g}$ to the $B^{1} \Sigma_{u}$ electron level. Subsequent de-excitation occurs initially to an excited vibrational level of the ground electron state with the emission of fluorescence radiation, and then down to the ground vibrational level through emission in the near-IR. Right: Reconstruction of the $\mathrm{H}_{2}$ fluorescence line profile observed towards TW Hya shows that $\mathrm{H}_{2}$ needs to be pre-conditioned to high temperatures prior to excitation (figure from Najita et al. 2007, from Protostars and Planets V by Reipurth et al. (c) 2007, the Arizona Board of Regents, reprinted by permission of the University of Arizona Press).

vibrational level in the ground electronic state, producing high energy radiation in the ultraviolet. Subsequent de-excitation from the excited vibrational state produces additional radiation in the nearIR. By reconstructing the the Lyman- $\alpha$ band from TW Hya, (Herczeg et al. 2004) showed that that $\mathrm{H}_{2}$ before the UV pumping needs to be very hot, residing in the innermost parts of the disk.

Acknowledgements The research leading to these results has received funding from the European Union Seventh Framework Programme FP7-2011 under grant agreement no 284405.

\section{References}

Bergin, E. A., Cleeves, L. I., Gorti, U., et al. 2013, Nature, 493, 644

Bik, A. \& Thi, W. F. 2004, A\&A, 427, L13

Brittain, S. D., Rettig, T. W., Simon, T., et al. 2003, ApJ, 588, 535 
EPJ Web of Conferences

Carmona, A., van der Plas, G., van den Ancker, M. E., et al. 2011, A\&A, 533, A39

Carr, J. S. \& Najita, J. R. 2008, Science, 319, 1504

Elitzur, M., Asensio Ramos, A., \& Ceccarelli, C. 2012, MNRAS, 422, 1394

Fedele, D., Bruderer, S., van Dishoeck, E. F., et al. 2013, A\&A, 559, A77

Goldsmith, P. F. \& Langer, W. D. 1999, ApJ, 517, 209

Guilloteau, S., Simon, M., Piétu, V., et al. 2014, A\&A, 567, A117

Herczeg, G. J., Wood, B. E., Linsky, J. L., Valenti, J. A., \& Johns-Krull, C. M. 2004, ApJ, 607, 369

Herzberg, G. W. 1950, Molecular spectra and molecular structure. Vol.1: Spectra of diatomic molecules, New York: Van Nostrand Reinhold, 1950, 2nd ed. (New York: Van Nostrand Reinhold, 1950, 2nd ed.)

Kamp, I. 2015a, in EPJ Web of Conferences, Vol. 102, Summer School on Protoplanetary Disks: Theory and Modeling Meet Observations, ed. I. Kamp, P. Woitke, \& J. D. Ilee

Kamp, I. 2015b, in EPJ Web of Conferences, Vol. 102, Summer School on Protoplanetary Disks: Theory and Modeling Meet Observations, ed. I. Kamp, P. Woitke, \& J. D. Ilee

Kóspál, Á., Moór, A., Juhász, A., et al. 2013, ApJ, 776, 77

Lahuis, F., van Dishoeck, E. F., Blake, G. A., et al. 2007, ApJ, 665, 492

Najita, J. R., Carr, J. S., Glassgold, A. E., \& Valenti, J. A. 2007, Protostars and Planets V, 507

Piétu, V., Dutrey, A., \& Guilloteau, S. 2007, A\&A, 467, 163

Rosenfeld, K. A., Andrews, S. M., Hughes, A. M., Wilner, D. J., \& Qi, C. 2013, ApJ, 774, 16

Salyk, C., Pontoppidan, K. M., Blake, G. A., et al. 2008, ApJL, 676, L49

Shaw, G., Ferland, G. J., Abel, N. P., Stancil, P. C., \& van Hoof, P. A. M. 2005, ApJ, 624, 794

Stahler, S. \& Palla, F. 2004, The Formation of Stars (WILEY-VCH Verlag GmbH \& Co. KGaA,Weinheim, ISBN: 3-527-40559-3)

Tennyson, J. 2005, Astronomical spectroscopy: an introduction to the atomic and molecular physics of astronomical spectra (Imperial College Press advanced physics texts, vol. 2, London, ISBN 1860945295)

Thi, W. F., Blake, G. A., van Dishoeck, E. F., et al. 2001, Nature, 409, 60

van der Plas, G., van den Ancker, M. E., Acke, B., et al. 2009, A\&A, 500, 1137 